\title{
Gaze through the clinical lens: molecular and clinical advancements of botanicals
}

\author{
Ammad A Farooqi ${ }^{1} \&$ Aamir Ahmad*,2 \\ ${ }^{1}$ Department of Molecular Oncology, Institute of Biomedical \& Genetic Engineering (IBGE), Islamabad, Pakistan \\ ${ }^{2}$ Department of Oncologic Sciences, Mitchell Cancer Institute, University of South Alabama, Mobile, AL 36604, USA \\ *Author for correspondence: aahmad@health.southalabama.edu

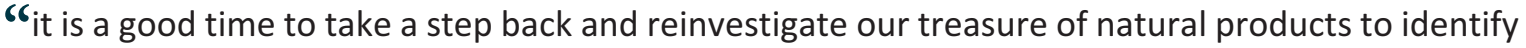 \\ botanicals with maximum efficacy, minimum off-target effects and excellent potential to \\ pleiotropically modulate signaling pathways in different cancers."
}

First draft submitted: 1 August 2018; Accepted for publication: 8 November 2018; Published online: 16 January 2019

Keywords: anticancer $\bullet$ bioavailability $\bullet$ botanicals $\bullet$ clinical trials $\bullet$ nutraceuticals $\bullet$ personalized nutrition

\section{Cancer therapy: the challenges}

Landmark discoveries and breakthroughs in cancer biology have sequentially shown that cancer is therapeutically challenging and rapidly accumulating experimental data have paradigmatically shifted our understanding about 'one size fits all' in cancer. It is being realized that overexpression of oncogenes; inactivation of tumor suppressors; deregulation of cell signaling pathways; tumor heterogeneity and the characteristically unique ability of cancer cells to resist drugs-associated toxicity are some of the mechanisms that underlie cancer onset and progression. Based on the insights gleaned from decades of research, it seems clear that cancer cells have the ability to rewire signaling cascades to survive under drug pressure. It is indeed alarming to see ever-emerging reports on the development of resistance against various drugs in different cancers. Despite promising results in xenograft mice models, many drugs fail to pass the tests of clinical trials. It is noteworthy that natural product research has enjoyed renaissance in the last decade or so and molecular biologists, pharmacologists and natural product researchers have paid more attention to the in-depth analysis of natural products. Therefore, it is a good time to take a step back and reinvestigate our treasure of natural products to identify botanicals with maximum efficacy, minimum off-target effects and excellent potential to pleiotropically modulate signaling pathways in different cancers.

\section{Anticancer agents of 'natural' origin}

There has always been a sharp divide between the proponents and the opponents of research on botanicals, natural agents or nutraceuticals. However, it needs to be recognized that almost half of all the US FDA-approved anticancer drugs have historically been natural products or their derivatives [1]. In addition, an overwhelming number of cancer patients often resort to taking dietary supplements of natural origin [2], based either on the traditional medicinal systems or the media coverage of preclinical studies. The continued efforts of researchers have resulted in testing of several of such putative anticancer compounds in clinical trials [3]. Information on completed as well as on-going clinical trials of some of these well-known anticancer compounds, such as curcumin, resveratrol, sulforaphane, epigallocatechin gallate (EGCG), lycopene, isoflavones, thymoquinone - among others, is easily available at ClinicalTrials.gov. Here we will discuss some recent and promising data emerging from clinical studies/trials.

\section{Emerging clinical data}

In a recent case study [4], Viscum album extract (VAE) administration was reported to cause $>50 \%$ regression of skull metastases from renal cancer, and the regression persisted for 2 years. Such skull metastases are resistant to radiotherapy and systemically administered therapies, hence why the VAE-associated therapeutic response is very promising. In another case study [5], VAE caused complete remission of lymph node metastases from metastatic 
malignant cutaneous melanoma and the patient was rendered tumor free. Even before these case studies, VAE was tested in a randomized Phase III study on late-stage pancreatic cancer patients. The first interim report [6] suggested marked improvement in median overall survival, with improvements in both 'good' and 'poor' prognosis subgroups of patients. Pancreatic tumors are especially difficult to treat and this progress underlines the enormous potential of botanical anticancer leads. One of the promises of such therapy is significantly reduced toxicity. It was not a surprise, therefore, that the Phase III study with VAE recognized its lack of toxicity [6]. A more recent observational clinical study further checked upon this property of VAE when nine out of 16 metastatic cancer patients receiving immune check inhibitors were simultaneously administered VAE [7]. No change in adverse effects was observed in VAE group. In view of these reports, more detailed studies need to be planned to fully understand the potential of $\mathrm{VAE}$ as a therapeutic agent versus an adjuvant therapy.

One of the biggest challenges with natural anticancer agents is their bioavailability, which has necessitated efforts to improve upon this drawback, resulting in the synthesis and testing of novel formulations [8]. In a recent clinical study [9], liposomes with elemene (a terpene found in celery, mint and other herbs) were reported to work effectively with whole-brain radiotherapy, resulting in prolonged nervous-system progression-free survival in non-small-cell lung cancer patients with multiple brain metastases. In a Phase II randomized, double-blind clinical trial, Polyphenon E (a green tea polyphenol formulation primarily consisting of EGCG) was noted to accumulate significantly in both nonmalignant and malignant bladder tissues [10], thus, confirming the potential of novel formulations in improving the bioavailability. Further confirmation was provided by a study wherein 12 breast cancer patients were administered greenselect phytosome (a lecithin formulation, enriched with catechin extracts) before they underwent surgery [11]. The repeated dosing, consisting of a daily dose for 4 weeks, resulted in an increase in total EGCG levels in plasma. Interestingly, total EGCG was detected in all tumor tissue samples and the median total EGCG concentrations were higher in the tumor tissues, as compared with the adjacently located normal tissues.

\section{Future perspective}

The studies discussed above are promising; however, one has to be objective in the overall analysis. For every clinical study with a positive outcome, there probably is another one with a neutral or even a negative outcome. For example, a randomized, placebo-controlled trial, involving daily intake of a catechin mixture for 1 year did not reduce the chances of prostate cancer in men with baseline atypical small acinar proliferation or high-grade, prostatic intraepithelial neoplasia [12]. Similarly, a placebo-controlled, double-blinded randomized pilot study conducted for evaluation of different natural products (resveratrol, turmeric and broccoli sprouts) found no statistically significant effect on prostate-specific antigen rise in men with biochemically recurrent prostate cancer [13]. Finally, muscadine grape skin extract (a pulverized muscadine grape skin that contains resveratrol, quercetin and ellagic acid) was found to increase PSA doubling time in patients with biochemically recurrent prostate cancer, but only in a subgroup of patients with SOD2 gene polymorphism [14]. Clinical studies are difficult to compare and those that are particularly challenging to conduct involve natural agents [2]. Therefore, it is best to learn from shortcomings of failed clinical trials and build upon the knowledge gained from the positive ones.

A lot of progress has been made toward a better understanding of signal transduction cascades that play a critical role in carcinogenesis and metastasis. However, the same cannot be said about the enormous potential of natural products, many of which are completely unexplored while others have not been extensively analyzed for their ability to therapeutically target oncogenic cell signaling pathways in specific cancer types or population groups. Furthermore, individual differences in the body's ability to metabolize and respond to nutrients are very important and gradually attracting considerable interest. This has led to the concept of personalized nutrition, where we will hopefully witness substantial progress in the next few years.

\section{Financial \& competing interests disclosure}

The authors have no relevant affiliations or financial involvement with any organization or entity with a financial interest in or financial conflict with the subject matter or materials discussed in the manuscript. This includes employment, consultancies, honoraria, stock ownership or options, expert testimony, grants or patents received or pending, or royalties.

No writing assistance was utilized in the production of this manuscript. 


\section{References}

1. Ahmad A. Expert commentary: dietary factors and natural product derivatives in cancer therapy. In: Nutraceuticals and Natural Product Derivatives: Disease Prevention \& Drug Discovery. Ullah M, Ahmad A (Eds.). Wiley-Blackwell, NJ, USA, 31-33 (2018).

2. Paller CJ, Denmeade SR, Carducci MA. Challenges of conducting clinical trials of natural products to combat cancer. Clin. Adv. Hematol. Oncol. 14(6), 447-455 (2016).

3. Li Y, Ahmad A, Kong D, Bao B, Sarkar FH. Recent progress on nutraceutical research in prostate cancer. Cancer Metastasis Rev. 33(2-3), 629-640 (2014).

4. Werthmann PG, Huber R, Kienle GS. Durable clinical remission of a skull metastasis under intralesional Viscum album extract therapy: case report. Head Neck 40(7), E77-E81 (2018).

5. Werthmann PG, Hintze A, Kienle GS. Complete remission and long-term survival of a patient with melanoma metastases treated with high-dose fever-inducing Viscum album extract: a case report. Medicine (Baltimore) 96(46), e8731 (2017).

6. Troger W, Galun D, Reif M, Schumann A, Stankovic N, Milicevic M. Viscum album [L.] extract therapy in patients with locally advanced or metastatic pancreatic cancer: a randomised clinical trial on overall survival. Eur. J. Cancer 49(18), 3788-3797 (2013).

7. Thronicke A, Steele ML, Grah C, Matthes B, Schad F. Clinical safety of combined therapy of immune checkpoint inhibitors and Viscum album L. therapy in patients with advanced or metastatic cancer. BMC Complement Altern. Med. 17(1), 534 (2017).

8. Siddiqui IA, Adhami VM, Bharali DJ et al. Introducing nanochemoprevention as a novel approach for cancer control: proof of principle with green tea polyphenol epigallocatechin-3-gallate. Cancer Res. 69(5), 1712-1716 (2009).

9. Sun YN, Zhang ZY, Zeng YC, Chi F, Jin XY, Wu R. Comparative efficacy of whole-brain radiotherapy with and without elemene liposomes in patients with multiple brain metastases from non-small-cell lung carcinoma. Curr. Oncol. 23(4), e377-e382 (2016).

10. Gee JR, Saltzstein DR, Kim K et al. A Phase II randomized, double-blind, presurgical trial of Polyphenon E in bladder cancer patients to evaluate pharmacodynamics and bladder tissue biomarkers. Cancer Prev. Res. (Phila.) 10(5), 298-307 (2017).

11. Lazzeroni M, Guerrieri-Gonzaga A, Gandini S et al. A presurgical study of lecithin formulation of green tea extract in women with early breast cancer. Cancer Prev. Res. (Phila.) 10(6), 363-370 (2017).

12. Kumar NB, Pow-Sang J, Egan KM et al. Randomized, placebo-controlled trial of green tea catechins for prostate cancer prevention. Cancer Prev. Res. (Phila.) 8(10), 879-887 (2015).

13. van Die MD, Williams SG, Emery J et al. A placebo-controlled double-blinded randomized pilot study of combination phytotherapy in biochemically recurrent prostate cancer. Prostate 77(7), 765-775 (2017).

14. Paller CJ, Zhou XC, Heath EI et al. Muscadine grape skin extract (MPX) in men with biochemically recurrent prostate cancer: a randomized, multicenter, placebo-controlled clinical trial. Clin. Cancer Res. 24(2), 306-315 (2018). 
